\title{
Towards a non-error floor multi-stream beamforming design for FBMC/OQAM
}

\author{
Màrius Caus*, Ana I. Perez-Neira ${ }^{\dagger *}$, Yao Cheng ${ }^{\ddagger}$ and Martin Haardt ${ }^{\ddagger}$ \\ ${ }^{*}$ Centre Tecnològic de Telecomunicacions de Catalunya (CTTC), Castelldefels, Barcelona, Spain \\ ${ }^{\dagger}$ Dept. of Signal Theory and Communications, Universitat Politècnica de Catalunya (UPC), Barcelona, Spain \\ ${ }^{\ddagger}$ Communications Research Laboratory, Ilmenau University of Technology, Ilmenau, Germany
}

\begin{abstract}
This paper investigates the application of filter bank multicarrier modulation based on the OQAM (FBMC/OQAM) to multiple-input-multiple-output (MIMO) systems. Existing solutions guarantee satisfactory performance when the streams multiplexed on each subcarrier $(S)$ and the number of transmit $\left(N_{T}\right)$ and receive $\left(N_{R}\right)$ antennas are related as $S=\min \left(N_{T}, N_{R}\right)$. When $S<\min \left(N_{T}, N_{R}\right)$, the techniques presented in previous works either exhibit an error floor or perform much worse than orthogonal frequency division multiplexing (OFDM). To make progress towards the combination of FBMC/OQAM with MIMO we propose a two-step approach and a coordinated beamforming algorithm to design the transmit and the receive processing. $\mathrm{Nu}-$ merical results show that the two-step method provides similar bit error rate (BER) as OFDM when $S+1=N_{T}=N_{R}$. Resorting to the coordinated beamforming solution, which is based on an iterative method, the application of FBMC/OQAM is extended to the general case $S<\min \left(N_{T}, N_{R}\right)$. Hence, the techniques presented in this paper demonstrate that FBMC/OQAM can achieve practically the same BER as OFDM with an increased spectral efficiency and a significantly decreased out-of-band radiation, which is an important advantage for non-contiguous spectrum allocations.
\end{abstract}

\section{INTRODUCTION}

The orthogonal frequency division multiplexing (OFDM) has been the modulation of choice in $4 \mathrm{G}$ cellular network standards. The main assets of OFDM are the ease of hardware implementation and the robustness against multipath fading. However, this comes with the price of degrading the spectral efficiency due to the cyclic prefix (CP) transmission. In addition, the large side lobes exhibited by the transmit signal make OFDM unsuitable when strict synchronization cannot be attained. The filter bank multicarrier modulation based on OQAM (FBMC/OQAM) is considered as an eligible candidate to substitute OFDM and become the air-interface of $5 \mathrm{G}$ networks [1]. The beauty of FBMC/OQAM is that subcarrier signals can be shaped with pulses that exhibit good time-frequency localization properties, while maximum bandwidth efficiency is achieved [2].

It is well-known that in presence of multipath fading the orthogonality between subcarriers in FBMC/OQAM is not preserved, which leads to inter-symbol interference (ISI) and inter-carrier interference (ICI). This has become the main obstacle to integrate FBMC/OQAM with multiple-inputmultiple-output (MIMO) communication systems [1]. The orthogonality issue is overcome by introducing the $\mathrm{CP}$ together with the adoption of the circular filtering [3], yet this entails wasting energy and losing spectral efficiency.

Previous works in the literature have concluded that if no $\mathrm{CP}$ is transmitted, then the MIMO techniques originally designed for OFDM can be successfully applied to FBMC/OQAM only in high coherence bandwidth channels [4]. The authors in [4] show that robustness against multipath fading is provided to a higher extent assuming that the channel frequency response (CFR) is flat at the subcarrier level, while it varies on adjacent subcarriers. However, satisfactory results are only guaranteed if $S=N_{R} \leq N_{T}$, where $S$ is the number of streams spatially multiplexed on each subcarrier and $N_{T}, N_{R}$ are respectively the number of transmit and receive antennas. Adopting the system model of [4], the iterative algorithm presented in [5] improves the results when $S<\min \left(N_{R}, N_{T}\right)$. Unfortunately the processing developed in [5] does not mitigate ICI, which yields an error floor in some multiantenna configurations. The technique proposed in [6] improves the resilience against the channel frequency selectivity with respect to [4], [5], since no assumptions are made about the flatness of the channel. Nevertheless, the number of variables required to represent the system model increases, which has an impact on the complexity. In addition, in some MIMO settings the solution addressed in [6] does not perform close to OFDM [7].

To overcome the limitations of existing techniques we propose two solutions: a two-step approach and a coordinated beamforming. In the two-step method, the precoders are first optimized to maximize the signal to leakage plus noise ratio (SLNR) given the equalizers. Then, equalizers are designed according to the minimum mean square error (MMSE) criterion fixing the precoders. The coordinated beamforming adopts similar SLNR-based and MMSE-based methods to respectively update the precoders and the equalizers iteratively. This algorithm is proposed to achieve competitive results in those MIMO settings where the two-step approach is not successful. The SLNR-based precoding has been previously proposed in FBMC/OQAM [8]. The main difference is that the work presented here considers multi-stream transmission and tackles the design of the equalizers. The contributions of this paper are summarized in the following.

- Simulation-based results show that the two-step approach remains competitive with OFDM and outperforms [4], [5] when $S+1=N_{R}=N_{T}$. Further, in those scenarios where the model adopted in [4] is valid and $S+1=N_{R}=N_{T}$, the performance provided by [6] 
and the two-step approach practically coincide. This is relevant because the notation of this paper is by far less complex than that considered in [6].

- At the expense of increasing the complexity, the coordinated beamforming generalizes the superiority of the SLNR-based and MMSE-based beamforming when $S<$ $\min \left(N_{R}, N_{T}\right)$. The experimental validation conducted in this paper reveals that the coordinated beamforming performs close to OFDM and clearly improves [4]-[6].

The rest of the paper is organized as follows. The next section defines the system model. Detailed descriptions of the two-step approach and the coordinated beamforming algorithm are provided in Sections III and IV, respectively. The numerical results are presented in Section $\mathrm{V}$ and finally the conclusions are drawn in Section VI.

\section{System MODEL}

This section provides the mathematical notation used to describe point-to-point MIMO communication systems that employ FBMC/OQAM. The transmitter and the receiver are equipped with $N_{T}$ and $N_{R}$ antennas, respectively. Borrowing the notation from [7], the interplay from the input of the synthesis filter bank to the output of the analysis filter bank on the $q$ th subcarrier can be expressed for $0 \leq q \leq M-1$ as

$$
\begin{aligned}
\check{\mathbf{d}}_{q}[k]= & \overline{\mathbf{A}}_{q}^{T} \check{\mathbf{H}}_{q} \overline{\mathbf{B}}_{q} \mathbf{d}_{q}[k]+\overline{\mathbf{A}}_{q}^{T} \overline{\mathbf{w}}_{q}[k] \\
& -\sum_{m=q-1}^{q+1} \overline{\mathbf{A}}_{q}^{T} \hat{\mathbf{H}}_{m} \overline{\mathbf{B}}_{m} \Im\left(\mathbf{i}_{q m}[k]\right) .
\end{aligned}
$$

In notation terms $\mathbf{d}_{q}[k]=\left[d_{q}^{1}[k] \ldots d_{q}^{S}[k]\right]^{T} \in \mathbb{R}^{S \times 1}$ represents the vector of PAM symbols that is multiplexed on the $q$ th subcarrier and the $k$ th time instant. The symbols are pre- and post-processed by the precoders $\left\{\mathbf{B}_{q}\right\} \in \mathbb{C}^{N_{T} \times S}$ and the equalizers $\left\{\mathbf{A}_{q}\right\} \in \mathbb{C}^{N_{R} \times S}$. The real-valued representation of the input/output relationship in (1) yields matrices $\overline{\mathbf{A}}_{q}=\left[\Re\left(\mathbf{A}_{q}^{T}\right) \Im\left(\mathbf{A}_{q}^{T}\right)\right]^{T}$ and $\overline{\mathbf{B}}_{q}=\left[\Re\left(\mathbf{B}_{q}^{T}\right) \Im\left(\mathbf{B}_{q}^{T}\right)\right]^{T}$. Let $\mathbf{w}_{q}[k] \in \mathbb{C}^{N_{R} \times 1}$ be the filtered noise that contaminates the reception of the $q$ th subcarrier. Hence, $\overline{\mathbf{w}}_{q}[k]=$ $\left[\Re\left(\mathbf{w}_{q}^{T}[k]\right) \Im\left(\mathbf{w}_{q}^{T}[k]\right)\right]^{T}$. The second line of (1) corresponds to ISI and ICI. The equivalent channel and interference channel matrices are defined as

$$
\begin{gathered}
\check{\mathbf{H}}_{m}=\left[\begin{array}{cc}
\Re\left(\mathbf{H}_{m}\right) & -\Im\left(\mathbf{H}_{m}\right) \\
\Im\left(\mathbf{H}_{m}\right) & \Re\left(\mathbf{H}_{m}\right)
\end{array}\right] \\
\hat{\mathbf{H}}_{m}=\left[\begin{array}{cc}
\Im\left(\mathbf{H}_{m}\right) & \Re\left(\mathbf{H}_{m}\right) \\
-\Re\left(\mathbf{H}_{m}\right) & \Im\left(\mathbf{H}_{m}\right)
\end{array}\right],
\end{gathered}
$$

where $\mathbf{H}_{m} \in \mathbb{C}^{N_{R} \times N_{T}}$ denotes the MIMO channel matrix evaluated on the radial frequency $\frac{2 \pi}{M} m$. If equalizers are constrained to only have in-phase components, then $\overline{\mathbf{A}}_{q}=$ $\mathbf{A}_{q} \in \mathbb{R}^{N_{R} \times S}$ and the augmented channel matrices become

$$
\begin{aligned}
\check{\mathbf{H}}_{m} & =\left[\begin{array}{ll}
\Re\left(\mathbf{H}_{m}\right) & -\Im\left(\mathbf{H}_{m}\right)
\end{array}\right] \\
\hat{\mathbf{H}}_{m} & =\left[\begin{array}{ll}
\Im\left(\mathbf{H}_{m}\right) & \Re\left(\mathbf{H}_{m}\right)
\end{array}\right] .
\end{aligned}
$$

The pure imaginary vector $\mathbf{i}_{q m}[k]$ is defined in [7]. Therein the statistical information is characterized when the prototype pulse is designed according to [9] with an overlapping factor equal to four. Then, $\mathbb{E}\left\{\mathbf{i}_{q m}[k] \mathbf{i}_{q m}^{H}[k]\right\}=E_{S} \sigma_{q m}^{2} \mathbf{I}_{S}$, where $\sigma_{q q-1}^{2}=\sigma_{q q+1}^{2}=0.1769$ and $\sigma_{q q}^{2}=0.646$ for all $q$. It is assumed that $\mathbb{E}\left\{\mathbf{d}_{q}[k] \mathbf{d}_{m}^{T}[n]\right\}=E_{S} \delta_{q, m} \delta_{k, n} \mathbf{I}_{S}$.

\section{TWO-STEP BEAMFORMING DESIGN}

The joint design of MIMO precoding and decoding matrices is investigated in this section. The approach that we propose to enhance the performance is divided into two steps. First, precoders are designed given the equalizers and then, the equalizers are updated fixing the precoders. Hence, only the MIMO decoding matrices have to be initialized.

\section{A. Design of the transmit processing}

To completely remove the interference the precoder can be designed to satisfy $\hat{\mathbf{H}}_{q} \overline{\mathbf{B}}_{q}=\mathbf{0}$. The zero forcing (ZF) approach only makes sense when equalizers are real-valued because the null space of (2) and (3) is the same, which implies that in the complex-valued counterpart the interference cannot be canceled without eliminating the desired signal. The drawback of projecting onto the null space of $\hat{\mathbf{H}}_{q}$ is that the spatial channel gains are dramatically reduced as if no projection was needed. The ZF only gives satisfactory performance if $S=$ $N_{R} \leq N_{T}$, as [4], [5], [7] show. The problem exhibited by the previous design is solved to some extent by the coordinated beamforming solution [5], by setting these new constraints $\overline{\mathbf{A}}_{q}^{T} \hat{\mathbf{H}}_{q} \overline{\mathbf{B}}_{q}=\mathbf{0}$ and forcing equalizers to be real-valued. Note that if an initial set of equalizers is assumed, the degrees of freedom after imposing the zero-interference constraint are increased from $2 N_{T}-N_{R}$ to $2 N_{T}-S$. The downside is that ISI is removed but ICI is not. The reason lies in the fact that in general we cannot assume that $\overline{\mathbf{A}}_{q-1}=\overline{\mathbf{A}}_{q}=\overline{\mathbf{A}}_{q+1}$ and, thus, $\overline{\mathbf{A}}_{m}^{T} \hat{\mathbf{H}}_{q} \overline{\mathbf{B}}_{q} \neq \mathbf{0}$ for $m=\{q-1, q+1\}$. As a consequence, the coordinated beamforming may not get rid of the ICI leading to an error floor. To achieve an effective interference cancellation without substantially worsening the spatial channel seen by the desired symbols, i.e., the diagonal elements of $\overline{\mathbf{A}}_{q}^{T} \check{\mathbf{H}}_{q} \overline{\mathbf{B}}_{q}$, we propose to resort to the SLNR. The motivation behind this choice is based on the results provided in [7], which confirm that the best strategy is not to cancel out the interference but it is enough to attenuate the unwanted signals $20 \mathrm{~dB}$ below the desired signal. Therefore, the SLNR-based precoder becomes an attractive solution, because unlike the coordinated beamforming in [5], we can control not only the ISI but the ICI as well. The metric to be optimized is formulated as

$$
\operatorname{SLNR}_{q}=\frac{\left\|\overline{\mathbf{A}}_{q}^{(0)^{T}} \check{\mathbf{H}}_{q} \overline{\mathbf{B}}_{q}\right\|_{F}^{2}}{\sum_{m=q-1}^{q+1} \sigma_{m q}^{2}\left\|\overline{\mathbf{A}}_{m}^{(0)^{T}} \hat{\mathbf{H}}_{q} \overline{\mathbf{B}}_{q}\right\|_{F}^{2}+\frac{N_{0}}{E_{s} 2}\left\|\overline{\mathbf{A}}_{q}^{(0)}\right\|_{F}^{2}} .
$$

Note that $\overline{\mathbf{A}}_{q}^{(0)}$ is fixed and is given beforehand. To get (6) we use the Frobenius norm $\|\mathbf{A}\|_{F}^{2}=\operatorname{tr}\left(\mathbf{A}^{T} \mathbf{A}\right)$, where $\operatorname{tr}($.$) is$ 
the trace operator. Concerning the contribution of the noise in the denominator, the following distribution has been assumed $\mathbf{w}_{q}[k] \sim \mathcal{C N}\left(\mathbf{0}, N_{0} \mathbf{I}_{N_{R}}\right)$. Let $\mathbf{I}_{N_{R}}$ be the $N_{R}$-dimensional identity matrix. If $\overline{\mathbf{A}}_{q}^{(0)} \in \mathbb{R}^{2 N_{R} \times S}$ it follows that the noise covariance matrix is $\mathbf{R}_{w_{q}}=\mathbb{E}\left\{\overline{\mathbf{w}}_{q}[k] \overline{\mathbf{w}}_{q}^{T}[k]\right\}=\frac{N_{0}}{2} \mathbf{I}_{2 N_{R}}$ and the channel matrices in (6) are given by (2) and (3). By contrast if $\overline{\mathbf{A}}_{q}^{(0)} \in \mathbb{R}^{N_{R} \times S}$, then $\mathbf{R}_{w_{q}}=\mathbb{E}\left\{\overline{\mathbf{w}}_{q}[k] \overline{\mathbf{w}}_{q}^{T}[k]\right\}=\frac{N_{0}}{2} \mathbf{I}_{N_{R}}$ and (4) and (5) should be plugged into (6). Bearing in mind this constraint $\left\|\overline{\mathbf{B}}_{q}\right\|_{F}^{2}=S$, the SLNR can be reformulated as

$$
\begin{gathered}
\operatorname{SLNR}_{q}=\frac{\operatorname{tr}\left(\overline{\mathbf{B}}_{q}^{T} \mathbf{D}_{q} \overline{\mathbf{B}}_{q}\right)}{\operatorname{tr}\left(\overline{\mathbf{B}}_{q}^{T} \mathbf{R}_{q} \overline{\mathbf{B}}_{q}\right)} \\
\mathbf{D}_{q}=\check{\mathbf{H}}_{q}^{T} \overline{\mathbf{A}}_{q}^{(0)} \overline{\mathbf{A}}_{q}^{(0)^{T}} \check{\mathbf{H}}_{q} \\
\mathbf{R}_{q}=\sum_{m=q-1}^{q+1} \sigma_{m q}^{2} \hat{\mathbf{H}}_{q}^{T} \overline{\mathbf{A}}_{m}^{(0)} \overline{\mathbf{A}}_{m}^{(0)^{T}} \hat{\mathbf{H}}_{q}+\frac{1}{S} \frac{N_{0}}{E_{s} 2}\left\|\overline{\mathbf{A}}_{q}^{(0)}\right\|_{F}^{2} \mathbf{I}_{2 N_{T}} .
\end{gathered}
$$

Now the optimization problem on each subcarrier becomes

$$
\begin{array}{cl}
\underset{\overline{\mathbf{B}}_{q}}{\operatorname{argmax}} & \mathrm{SLNR}_{q} \\
\text { s.t. } & \left\|\overline{\mathbf{B}}_{q}\right\|_{F}^{2}=S \\
& \overline{\mathbf{B}}_{q}^{T} \mathbf{D}_{q} \overline{\mathbf{B}}_{q} \rightarrow \text { diagonal. }
\end{array}
$$

Forcing $\overline{\mathbf{B}}_{q}^{T} \mathbf{D}_{q} \overline{\mathbf{B}}_{q}$ to have a diagonal structure allows us to benefit from the theory developed in [10]. Then, the solution of (10) is obtained by computing the generalized singular value decomposition of the matrix pair $\left\{\mathbf{D}_{q}, \mathbf{R}_{q}\right\}$. Actually, the optimal solution consists in setting $\overline{\mathbf{B}}_{q}=\left[\mathbf{t}_{1 q} \ldots \mathbf{t}_{S q}\right] \in \mathbb{R}^{2 N_{T} \times S}$, where $\mathbf{t}_{i q}$ corresponds to the singular vector associated to the $i$ th dominant singular value. To fulfill the power constraint the precoders are properly scaled so that its columns have unit norm. Then, the power is uniformly distributed among subcarriers as follows: $\mathbb{E}\left\{\left\|\mathbf{B}_{q} \mathbf{d}_{q}[k]\right\|_{F}^{2}\right\}=E_{S}\left\|\mathbf{B}_{q}\right\|_{F}^{2}=$ $E_{S}\left\|\overline{\mathbf{B}}_{q}\right\|_{F}^{2}=S E_{S}$. The design of power allocation algorithms is left for future work.

\section{B. Design of the receive processing}

The objective of the second step is to refine the design of $\overline{\mathbf{A}}_{q}^{(0)}$ setting the precoders according to previous section. The updated receive beamformers are obtained by minimizing the mean square error (MSE), i.e.,

$$
\underset{\overline{\mathbf{A}}_{q}}{\operatorname{argmin}} \mathrm{MSE}_{q},
$$

with

$$
\begin{aligned}
\operatorname{MSE}_{q}= & \mathbb{E}\left\{\left\|\check{\mathbf{d}}_{q}[k]-\mathbf{d}_{q}[k]\right\|^{2}\right\}=E_{S} \operatorname{tr}\left(\mathbf{I}_{S}-2 \overline{\mathbf{A}}_{q}^{T} \check{\mathbf{H}}_{q} \overline{\mathbf{B}}_{q}\right) \\
& +\operatorname{tr}\left(\overline{\mathbf{A}}_{q}^{T}\left(\sum_{m=q-1}^{q+1} E_{S} \sigma_{q m}^{2} \hat{\mathbf{H}}_{m} \overline{\mathbf{B}}_{m} \overline{\mathbf{B}}_{m}^{T} \hat{\mathbf{H}}_{m}^{T}\right) \overline{\mathbf{A}}_{q}\right) \\
& +\operatorname{tr}\left(\overline{\mathbf{A}}_{q}^{T}\left(E_{S} \check{\mathbf{H}}_{q} \overline{\mathbf{B}}_{q} \overline{\mathbf{B}}_{q}^{T} \check{\mathbf{H}}_{q}^{T}+\mathbf{R}_{w_{q}}\right) \overline{\mathbf{A}}_{q}\right) .
\end{aligned}
$$

The solution of (11) is known and can be expressed as

$$
\begin{aligned}
\overline{\mathbf{A}}_{q}= & \left(\sum_{m=q-1}^{q+1} \sigma_{q m}^{2} \hat{\mathbf{H}}_{m} \overline{\mathbf{B}}_{m} \overline{\mathbf{B}}_{m}^{T} \hat{\mathbf{H}}_{m}^{T}\right. \\
& \left.+\check{\mathbf{H}}_{q} \overline{\mathbf{B}}_{q} \overline{\mathbf{B}}_{q}^{T} \check{\mathbf{H}}_{q}^{T}+\frac{1}{E_{S}} \mathbf{R}_{w_{q}}\right)^{-1} \check{\mathbf{H}}_{q} \overline{\mathbf{B}}_{q} .
\end{aligned}
$$

It is worth mentioning that $\overline{\mathbf{A}}_{q}$ can be complex-valued regardless whether $\overline{\mathbf{A}}_{q}^{(0)}$ is real- or complex-valued. Depending on the case either (2),(3) or (4),(5) is used when defining $\overline{\mathbf{A}}_{q}$.

\section{Initial design}

The SLNR-based precoding design described in Section III-A hinges on the initialization of the MIMO decoding matrices. Hence, the initial receive processing has to be judiciously selected. The iterative algorithm described in [5] sets $\overline{\mathbf{A}}_{q}^{(0)}=$ $\left[\mathbf{u}_{1 q} \ldots \mathbf{u}_{S q}\right]$, where $\mathbf{u}_{i q}$ corresponds to the left singular vector of $\check{\mathbf{H}}_{q} \mathbf{V}_{q}^{0}$ associated with the $i$ th dominant singular value, i.e. $\lambda_{i q}$. The columns of $\mathbf{V}_{q}^{0} \in \mathbb{R}^{2 N_{T} \times 2 N_{T}-N_{R}}$ span the null space of $\hat{\mathbf{H}}_{q}$ and are selected so that $\left(\mathbf{V}_{q}^{0}\right)^{T} \mathbf{V}_{q}^{0}=\mathbf{I}_{2 N_{T}-N_{R}}$. This design imposes the use of (4),(5) and $2 N_{T}-N_{R} \geq S$. The same strategy cannot be followed in the complex case because (2) and (3) span the same space and, as a consequence $\check{\mathbf{H}}_{q} \mathbf{V}_{q}^{0}=\mathbf{0}$ when $N_{T}>N_{R}$. Sticking to the case where equalizers are real-valued, this section aims at justifying the use of $\left\{\mathbf{u}_{1 q} \ldots \mathbf{u}_{S q}\right\}$ to build the receive beamformers. The idea is to jointly design the equalizers and the precoders. Unfortunately, the problem is intricate and the joint optimization is definitely complex. To relax the original problem we propose to follow the two-step approach. That is, first precoders are designed and then, in the second step equalizers are optimized. The main difference with respect to III-A is that now we cannot rely on any initial design when addressing the design of the precoders. In order to simplify the problem we assume that $\overline{\mathbf{A}}_{q-1}^{(0)}=\overline{\mathbf{A}}_{q}^{(0)}=\overline{\mathbf{A}}_{q+1}^{(0)}$ when the $q$ th subcarrier is addressed. If the channel frequency selectivity is not sever, the approximation does not excessively deviate from the exact expression. Then, the SLNR can be written as

$$
\operatorname{SLNR}_{q}=\frac{\left\|\overline{\mathbf{A}}_{q}^{(0)^{T}} \check{\mathbf{H}}_{q} \overline{\mathbf{B}}_{q}^{(0)}\right\|_{F}^{2}}{\left\|\overline{\mathbf{A}}_{q}^{(0)^{T}} \hat{\mathbf{H}}_{q} \overline{\mathbf{B}}_{q}^{(0)}\right\|_{F}^{2}+\frac{N_{0}}{E_{s} 2}\left\|\overline{\mathbf{A}}_{q}^{(0)}\right\|_{F}^{2}} .
$$

To get (14) we have taken into account that $\sigma_{m q-1}^{2}+\sigma_{m q}^{2}+$ $\sigma_{m q+1}^{2} \approx 1$. With (14) the problem on each subcarrier can be independently treated, but it is still difficult to find a closedform solution. To further alleviate the complexity we relax the SLNR maximization and instead we focus on the lower bound:

$$
\mathrm{LB}_{q}=\frac{\lambda_{N_{R}}\left(\overline{\mathbf{A}}_{q}^{(0)} \overline{\mathbf{A}}_{q}^{(0)^{T}}\right)}{\left\|\overline{\mathbf{A}}_{q}^{(0)}\right\|_{F}^{2}} \frac{\left\|\check{\mathbf{H}}_{q} \overline{\mathbf{B}}_{q}^{(0)}\right\|_{F}^{2}}{\left\|\hat{\mathbf{H}}_{q} \overline{\mathbf{B}}_{q}^{(0)}\right\|_{F}^{2}+\frac{N_{0}}{E_{s} 2}} .
$$

Let $\lambda_{i}(\mathbf{A})$ be the $i$ th largest singular value of the matrix $\mathbf{A}$. To compute the metric $\mathrm{LB}_{q}$ it is important to remark that $\lambda_{N}(\mathbf{A}) \operatorname{tr}(\mathbf{B}) \leq \operatorname{tr}(\mathbf{A B}) \leq \lambda_{1}(\mathbf{A}) \operatorname{tr}(\mathbf{B})$, for symmetric matrix $\mathbf{A} \in \mathbb{R}^{N \times N}$ and positive-semidefinite matrix $\mathbf{B} \in \mathbb{R}^{N \times N}$ [11]. 
Using the first and the second inequality, respectively, in the numerator and the denominator of (14), it can be verified that $\mathrm{SLNR}_{q} \geq \mathrm{LB}_{q}$. It is worth mentioning that $\mathrm{LB}_{q}=0$ if $S<N_{R}$. Therefore, from here onwards we focus on the case $S=N_{R}$ to derive the initial design. Then, note that the more spread out are the singular values of $\overline{\mathbf{A}}_{q}^{(0)} \overline{\mathbf{A}}_{q}^{(0)^{T}}$, the looser is the bound. To tighten the bound the singular values should be equal, which translates into this constraint $\overline{\mathbf{A}}_{q}^{(0)^{T}} \overline{\mathbf{A}}_{q}^{(0)}=\mathbf{I}_{S}$. Then, $\mathrm{LB}_{q}$ does not depend on the equalizer and the optimization problem becomes

$$
\begin{aligned}
\underset{\overline{\mathbf{B}}_{q}}{\operatorname{argmax}} & \mathrm{LB}_{q} \\
\text { s.t. } & \left\|\overline{\mathbf{B}}_{q}^{(0)}\right\|_{F}^{2}=S, \quad \overline{\mathbf{A}}_{q}^{(0)^{T}} \overline{\mathbf{A}}_{q}^{(0)}=\mathbf{I}_{S} \\
& \overline{\mathbf{B}}_{q}^{(0)^{T}} \check{\mathbf{H}}_{q}^{T} \check{\mathbf{H}}_{q} \overline{\mathbf{B}}_{q}^{(0)} \rightarrow \text { diagonal. }
\end{aligned}
$$

According to [10], the solution of (16) is such that: i) $\overline{\mathbf{B}}_{q}^{(0)^{T}}\left(\hat{\mathbf{H}}_{q}^{T} \hat{\mathbf{H}}_{q}+\frac{1}{S} \frac{N_{0}}{E_{s} 2} \mathbf{I}_{2 N_{T}}\right) \overline{\mathbf{B}}_{q}^{(0)}$ must be a diagonal matrix with equal entries and ii) $\overline{\mathbf{B}}_{q}^{(0)^{T}} \check{\mathbf{H}}_{q}^{T} \check{\mathbf{H}}_{q} \overline{\mathbf{B}}_{q}^{(0)}$ has to exhibit a diagonal structure. Hence, the precoder is obtained by computing the $S$ dominant generalized singular vectors of this pair $\left(\check{\mathbf{H}}_{q}^{T} \check{\mathbf{H}}_{q}, \hat{\mathbf{H}}_{q}^{T} \hat{\mathbf{H}}_{q}+\frac{1}{S} \frac{N_{0}}{E_{s} 2} \mathbf{I}_{2 N_{T}}\right)$. Based on the requirements i) and ii), the precoder that solves (16) is given by

$$
\overline{\mathbf{B}}_{q}^{(0)}=\left[\mathbf{V}_{q}^{0} \mathbf{b}_{1 q} \ldots \mathbf{V}_{q}^{0} \mathbf{b}_{S q}\right]
$$

if the generalized singular vectors are orthogonal. The column vectors $\left\{\mathbf{b}_{1 q} \ldots \mathbf{b}_{S q}\right\}$ correspond to the $S$ first right singular vectors of $\tilde{\mathbf{H}}_{q} \mathbf{V}_{q}^{0}$. The fulfillment of i) and ii) can be readily verified recalling that the matrix $\mathbf{V}_{q}^{0}$ satisfies $\hat{\mathbf{H}}_{q} \mathbf{V}_{q}^{0}=\mathbf{0}$ and $\left(\mathbf{V}_{q}^{0}\right)^{T} \mathbf{V}_{q}^{0}=\mathbf{I}_{2 N_{T}-N_{R}}$. It must be mentioned that in general we cannot assume that the columns of the precoder that solve (16) are orthogonal, which may compromise the validity of (17). The numerical results provided in Section $\mathrm{V}$ reveal that (17) is reasonably close to the exact value. Accepting (17) as the true expression, the input/output relation becomes

$$
\check{\mathbf{d}}_{q}[k]=\overline{\mathbf{A}}_{q}^{(0)^{T}}\left(\sum_{i=1}^{S} \lambda_{i q} \mathbf{u}_{i q}\right) \mathbf{d}_{q}[k]+\overline{\mathbf{A}}_{q}^{(0)^{T}} \overline{\mathbf{w}}_{q}[k] .
$$

Note that ISI and ICI terms are canceled out because $\hat{\mathbf{H}}_{q} \mathbf{V}_{q}^{0}=$ 0. In addition, the noise is not enhanced because we impose $\overline{\mathbf{A}}_{q}^{(0)^{T}} \overline{\mathbf{A}}_{q}^{(0)}=\mathbf{I}_{S}$. In the light of previous observations MIMO decoding matrices are designed to maximize the energy of the desired signal. Hence, the problem is posed as follows:

$$
\begin{aligned}
\underset{\overline{\mathbf{A}}_{q}^{(0)}}{\operatorname{argmax}} & \left\|\sum_{i=1}^{S} \lambda_{i q} \mathbf{u}_{i q}^{T} \overline{\mathbf{A}}_{q}^{(0)}\right\|_{F}^{2} \\
\text { s.t. } & \overline{\mathbf{A}}_{q}^{(0)^{T}} \overline{\mathbf{A}}_{q}^{(0)}=\mathbf{I}_{S} .
\end{aligned}
$$

Since $\mathbf{u}_{i q}^{T} \mathbf{u}_{j q}=\delta_{i, j}$, the best strategy to maximize the objective function is to set $\overline{\mathbf{A}}_{q}^{(0)}=\left[\mathbf{u}_{1 q} \ldots \mathbf{u}_{S q}\right]$. With this design, it can be verified that $\mathrm{LB}_{q}=\mathrm{SLNR}_{q}$. To sum up, this section shows that the equalizer that maximizes $\mathrm{LB}_{q}$ when $S=N_{R}$ is reasonably close to the first $S$ left singular vectors of $\check{\mathbf{H}}_{q} \mathbf{V}_{q}^{0}$. For $S<N_{R}$ we have observed promising results by following the same approach but replacing $\lambda_{N_{R}}\left(\overline{\mathbf{A}}_{q}^{(0)} \overline{\mathbf{A}}_{q}^{(0)^{T}}\right)$ with $\lambda_{S}\left(\overline{\mathbf{A}}_{q}^{(0)} \overline{\mathbf{A}}_{q}^{(0)^{T}}\right)$ in (15). Then, we can claim that the gap due to the SLNR relaxation is zero as well, if (17) and (19) are used to design the matrix pair $\left\{\overline{\mathbf{A}}_{q}^{(0)}, \overline{\mathbf{B}}_{q}^{(0)}\right\}$.

\section{COORDINATED BEAMFORMING DESIGN}

In this section, we propose a new coordinated beamforming design. This scheme is able to achieve a superior performance over the two-step design. The application of the coordinated beamforming concept in MIMO FBMC/OQAM systems was first addressed in [5]. Although the coordinated beamforming technique in [5] alleviates the dimensionality constraint that $N_{R}$ must not exceed $N_{T}$, it still suffers from a performance degradation due to residual ICI. To achieve a more effective mitigation of both the ISI and ICI, we propose to incorporate the SLNR-based transmit processing introduced similarly as in Section III-A into the coordinated beamforming design. Similarly as [5], the precoding matrices and the decoding matrices are computed jointly and iteratively. It is worth mentioning that the proposed coordinated beamforming design restricts the MIMO decoding matrices to be real-valued, thus the system model is defined by (4) and (5). At the end of this section it is justified why the complex-valued counterpart has been discarded. Note that when we stick to the real case and $\left(2 N_{T}-N_{R}\right) \geq S$ the initialization of the decoding matrices employed in the two-step beamforming design is adopted. Otherwise, the decoding matrices are initialized randomly. We summarize the proposed coordinated beamforming algorithm as follows:

- Step 1: Initialize the real-valued decoding matrices $\overline{\mathbf{A}}_{q}^{(0)} \in \mathbb{R}^{N_{R} \times S}$ for all the subcarriers, set the iteration index $p$ to zero, and set a threshold $\epsilon$ for the stopping criterion.

- Step 2: Set $p \rightarrow p+1$ and compute the precoding matrices $\mathbf{B}_{q}^{(p)}$ for all the subcarriers based on the SLNR-based beamforming design described in Section III-A. The columns of $\overline{\mathbf{B}}_{q}^{(p)}$ correspond to the generalized singular vectors associated with the $S$ largest generalized singular values of the pair $\left\{\mathbf{D}_{q}^{(p)}, \mathbf{R}_{q}^{(p)}\right\}$, where

$$
\begin{aligned}
& \mathbf{D}_{q}^{(p)}=\check{\mathbf{H}}_{q}^{T} \overline{\mathbf{A}}_{q}^{(p-1)} \overline{\mathbf{A}}_{q}^{(p-1)^{T}} \check{\mathbf{H}}_{q}, \\
\mathbf{R}_{q}^{(p)}= & \sum_{m=q-1}^{q+1} \sigma_{m q}^{2} \hat{\mathbf{H}}_{q}^{T} \overline{\mathbf{A}}_{m}^{(p-1)} \overline{\mathbf{A}}_{m}^{(p-1)^{T}} \hat{\mathbf{H}}_{q}+ \\
& \frac{1}{S} \frac{N_{0}}{E_{s} 2}\left\|\overline{\mathbf{A}}_{q}^{(p-1)}\right\|_{F}^{2} \mathbf{I}_{2 N_{T}} .
\end{aligned}
$$

The precoding matrices are normalized such as the transmit power constraint is fulfilled.

- Step 3: Update the decoding matrices $\overline{\mathbf{A}}_{q}^{(p)}$ for all the subcarriers. The decoding matrices that minimize the 
MSE adopt this form

$$
\begin{aligned}
\overline{\mathbf{A}}_{q}^{(p)}= & \left(\sum_{m=q-1}^{q+1} \sigma_{q m}^{2} \hat{\mathbf{H}}_{m} \overline{\mathbf{B}}_{m}^{(p)} \overline{\mathbf{B}}_{m}^{(p)^{T}} \hat{\mathbf{H}}_{m}^{T}+\right. \\
& \left.\check{\mathbf{H}}_{q} \overline{\mathbf{B}}_{q}^{(p)} \overline{\mathbf{B}}_{q}^{(p)^{T}} \check{\mathbf{H}}_{q}^{T}+\frac{\mathbf{R}_{w_{q}}}{E_{S}}\right)^{-1} \check{\mathbf{H}}_{q} \overline{\mathbf{B}}_{q}^{(p)} .
\end{aligned}
$$

- Step 4: Track the variation of the residual interference to determine the termination of the algorithm. The following term that provides a measure of the inter-stream interference, the ISI, and the ICI at the $p$ th iteration can be defined

$$
\begin{aligned}
\xi^{(p)}= & \frac{1}{M} \sum_{q=0}^{M-1}\left\|\operatorname{off}\left(\overline{\mathbf{A}}_{q}^{(p)^{T}} \check{\mathbf{H}}_{q} \overline{\mathbf{B}}_{q}^{(p)}\right)\right\|_{F}^{2}+ \\
& \frac{1}{M} \sum_{q=0}^{M-1} \sum_{m=q-1}^{q+1} \sigma_{q m}^{2}\left\|\overline{\mathbf{A}}_{q}^{(p)^{T}} \hat{\mathbf{H}}_{m} \overline{\mathbf{B}}_{m}^{(p)}\right\|_{F}^{2},
\end{aligned}
$$

where off $(\cdot)$ denotes an operation of replacing all elements on the diagonal of the input matrix by zeros. Then, we approximate the slope of the variation of the residual interference via [7]

$$
\xi^{(p)^{\prime}}=\frac{\left|\xi^{(p)}-\xi^{(p-1)}\right|}{\xi^{(p-1)}} .
$$

If $\xi^{(p)^{\prime}}<\epsilon$, terminate the iterative procedure. Otherwise go back to Step 2 and further update the precoding matrices and decoding matrices. Alternatively, a stopping criterion based on the change of the precoding matrices [5] can also be employed.

It is worth noting that a joint-subcarrier processing is involved, i.e., the precoding matrices and decoding matrices for all the subcarriers are updated jointly. By contrast, the coordinated beamforming algorithm in [5] is on a per-subcarrier basis. The lack of the joint-subcarrier processing leads to its failure of effectively mitigating the ICI. In addition, we have observed that rendering the decoding matrices as complexvalued in this coordinated beamforming algorithm results in a much worse performance compared to the real-valued design described above. On the one hand, adopting complex-valued decoding matrices keeps the full degrees of freedom. On the other hand, it becomes even more challenging to suppress the interference in both the real domain and the imaginary domain. As the impact of the latter consequence overweighs that of the former, the performance is degraded compared to the realvalued design of the decoding matrices.

\section{NUMERICAL RESULTS}

To assess the performance of the proposed techniques the bit error rate (BER) is evaluated in this section. Regarding the system parameters, the bandwidth is $B=10 \mathrm{MHz}$, the sampling frequency is $f_{s}=11.2 \mathrm{MHz}$ and the number of subcarriers is $M=1024$. The propagation conditions are generated according to the ITU Vehicular A channel model [12], so that (1) is valid. The symbols are drawn from the 4-PAM constellation, which means that the entries of the
Table I

EVALUATION OF $\eta$ WITH A MIMO $4 \times 4 \times 3$ CONFIGURATION.

\begin{tabular}{|c|c|c|c|c|c|}
\hline$\frac{E_{S}}{N_{0}}(\mathrm{~dB})$ & 0 & 5 & 10 & 15 & 20 \\
\hline$\eta$ & $1.59 \%$ & $0.77 \%$ & $0.31 \%$ & $0.11 \%$ & $3.55 \times 10^{-2} \%$ \\
\hline
\end{tabular}

vectors $\left\{\mathbf{d}_{q}[k]\right\}$ can be obtained by taking either the real or the imaginary parts of 16-QAM symbols. The two-step approach and the coordinated beamforming, which are respectively identified with the acronyms TS and CBF, are confronted with the techniques presented in [4]-[6]. To carry out a fair comparison the power is uniformly distributed in [4], [6].

It is worth highlighting that the algorithm described in Section III is initialized by setting the receive beamformers as it is proposed in Section III-C. As for the receive processing, we opt to use the expression (13) with all the degrees of freedom. In other words, the equalizers are complex-valued. Unlike the coordinated beamforming algorithm, there is no penalty for using complex-valued MIMO decoding matrices in the non-iterative two step approach.

OFDM is included in the comparison as well because it is the dominant technology nowadays. In this case the transmit and the receive beamformers are designed to diagonalize the channel as proposed in [13], but without optimizing the power distribution. It is worth recalling that in the OFDM context the subcarrier signals convey 16-QAM symbols with a symbol rate twice that of FBMC/OQAM systems. Due to the $\mathrm{CP}$ transmission this percentage of energy $\left(1+L_{C P} / M\right)$ is wasted, where $L_{C P}$ accounts for the length of the CP.

The reduced out-of-band radiation of FBMC/OQAM has been harnessed to increase the number of active carriers from $M_{a}=720$, which is the standard value used by OFDM, to $M_{a}=756$ [4]. Hence, the spectral efficiency values are in bits/s/Hz: $($ FBMC/OQAM $)=3.3075 \times S$ and $(\mathrm{OFDM})=2.8 \times S$, for $L_{C P}=\mathrm{M} / 8$.

To define the multiantenna configuration in the simulations the following compact notation is used: $N_{T} \times N_{R} \times S$. Before evaluating the two-step approach, it is necessary to know if (17) has moved away from the precoder that maximizes $\mathrm{LB}_{q}$. Let $\mathrm{LB}_{q}^{\text {opt }}$ denote the solution of (16) and $\mathrm{LB}_{q}$ be the ratio when (17) is plugged into (15). In order to gain insight into the reliability of the approximation made in (17), the following magnitude is defined

$$
\eta=\frac{1}{M} \sum_{q=0}^{M-1} \frac{\left|\mathrm{LB}_{q}^{o p t}-\mathrm{LB}_{q}\right|}{\mathrm{LB}_{q}^{\text {opt }}} .
$$

The values gathered in Table I are sufficiently small to conclude that the difference is almost negligible.

As Figure 1 highlights the technique addressed in [5] exhibits an error floor. The ZF described in [4] performs poorly when $S<N_{R}$ confirming the results provided in [5], [7]. The TS and the solution presented in [6] practically achieve the same BER slightly outperforming OFDM at low and moderate $\frac{E_{S}}{N_{0}}$. Nevertheless, it is important to remark that the expressions involved in the definition of the system model are much more 


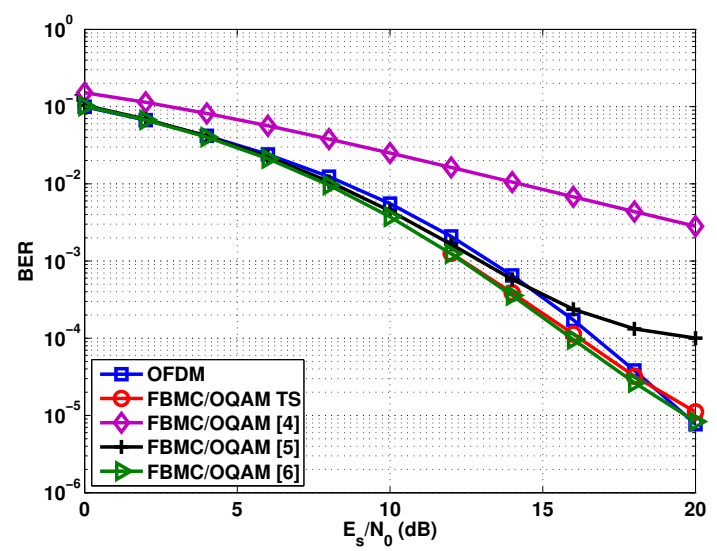

Figure 1. BER vs. $\frac{E_{S}}{N_{0}}$ in OFDM and FBMC/OQAM systems with a MIMO $4 \times 4 \times 3$ configuration.

complex in [6] than those presented in Section II. Similar results have been observed in the $3 \times 3 \times 2$ configuration, but due to space limitations the BER plot is not included.

To evaluate the performance of the coordinated beamforming algorithm, we now consider a $4 \times 4 \times 2$ MIMO setting. We adopt the stopping criterion with respect to (24) and set the threshold $\epsilon=10^{-2}$. In Figure 2 it can be observed that compared to the technique in [5], the proposed coordinated beamforming algorithm achieves a significant improvement performing close to OFDM thanks to the more effective suppression of the ICI. Note that in Figure 2 the TS and the solutions addressed in [4], [6] fail to achieve competitive results. The good results exhibited by the algorithm described in Section IV also hold true in the $6 \times 7 \times 5$ setting, but due to the lack of space the corresponding figure is not depicted.

\section{CONCLUSion}

This paper tackles MIMO transmitter and receiver designs for FBMC/OQAM systems. Precoders and equalizers are driven by the SLNR and the MMSE metrics, respectively. In the MIMO setting $S+1=N_{T}=N_{R}$ the first strategy, which is based on a two-step approach, achieves almost the same BER as OFDM and outperforms state-of-the-art schemes using a simplified notation that assumes flat fading conditions at the subcarrier level. To extend the good results for the general case $S<\min \left(N_{T}, N_{R}\right)$ an iterative algorithm is devised, where the transmit and the receive beamformers are governed by the SLNR and the MMSE, respectively. Simulation-based results reveal that the iterative algorithm shows superior performance when compared to existing methods and remains competitive with OFDM. The work elaborated in this paper confirms that FBMC/OQAM can benefit from the spatial diversity to improve the link reliability and the spectral efficiency.

\section{ACKNOWLEDGMENT}

This work has received funding from the Spanish Ministry of Economy and Competitiveness (Ministerio de Economia y Competitividad) under project TEC2011-29006-C03-02 (GRE3N-LINKMAC), from the Catalan Government under grant 2014SGR1567 and

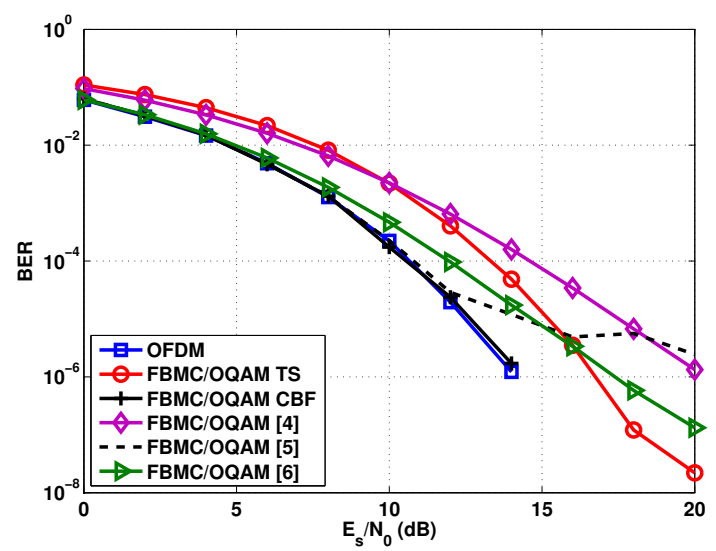

Figure 2. BER vs. $\frac{E_{S}}{N_{0}}$ in OFDM and FBMC/OQAM systems with a MIMO $4 \times 4 \times 2$ configuration.

from the European Commission through the EMPhAtiC project (ICT318362).

\section{REFERENCES}

[1] M. Schellmann, Z. Zhao, H. Lin, P. Siohan, N. Rajatheva, V. Luecken, and A. Ishaque, "FBMC-based air interface for 5G mobile: Challenges and proposed solutions," in 2014 9th International Conference on Cognitive Radio Oriented Wireless Networks and Communications (CROWNCOM), June 2014, pp. 102-107.

[2] P. Siohan, C. Siclet, and N. Lacaille, "Analysis and design of OFDM/OQAM systems based on filterbank theory," IEEE Transactions on Signal Processing, vol. 50, no. 5, pp. 1170 -1183, may 2002.

[3] H. Lin and P. Siohan, "An advanced multi-carrier modulation for future radio systems," in 2014 IEEE International Conference on Acoustics, Speech and Signal Processing (ICASSP), May 2014, pp. 8097-8101.

[4] M. Caus and A. I. Perez-Neira, "Multi-stream transmission in MIMOFBMC systems," in 2013 IEEE International Conference on Acoustics, Speech and Signal Processing, may 2013, pp. 5041-5045.

[5] Y. Cheng, P. Li, and M. Haardt, "Coordinated beamforming in MIMO FBMC/OQAM systems," in 2014 IEEE International Conference on Acoustics, Speech and Signal Processing, May 2014, pp. 484-488.

[6] M. Caus and A. I. Perez-Neira, "Multi-Stream Transmission for Highly Frequency Selective Channels in MIMO-FBMC/OQAM Systems," IEEE Transactions on Signal Processing, vol. 62, no. 4, pp. 786-796, Feb 2014.

[7] - "Experimental performance bounds of MIMO-FBMC/OQAM systems," in Proceedings of European Wireless 2014; 20th European Wireless Conference, May 2014, pp. 1-6.

[8] U. Jayasinghe, N. Rajatheva, and M. Latva-aho, "Application of a leakage based precoding scheme to mitigate intrinsic interference in FBMC," in 2013 IEEE International Conference on Communications (ICC), June 2013, pp. 5268-5272.

[9] M. Bellanger, "Specification and design of a prototype filter for filter bank based multicarrier transmission," in 2001 IEEE International Conference on Acoustics, Speech, and Signal Processing, vol. 4, 2001, pp. 2417-2420.

[10] M. Sadek, A. Tarighat, and A. Sayed, "A Leakage-Based Precoding Scheme for Downlink Multi-User MIMO Channels," IEEE Transactions on Wireless Communications, vol. 6, no. 5, pp. 1711-1721, May 2007.

[11] S.-D. Wang, T.-S. Kuo, and C.-F. Hsu, "Trace bounds on the solution of the algebraic matrix Riccati and Lyapunov equation," IEEE Transactions on Automatic Control, vol. 31, no. 7, pp. 654-656, Jul 1986.

[12] "Guidelines for the evaluation of radio transmission technologies for IMT-2000," in Recommendation ITU-R M.1225, 1997.

[13] D. Palomar, J. Cioffi, and M. Lagunas, "Joint Tx-Rx beamforming design for multicarrier MIMO channels: a unified framework for convex optimization," IEEE Transactions on Signal Processing, vol. 51, no. 9, pp. $2381-2401$, sept. 2003. 